\title{
Extremes makeover
}

\author{
More frequent and graver extreme events continue to remind us of the perils of climate change, but systematic \\ consideration of these extremes in energy systems studies and full accounting of their impacts remains a challenge.
}

( limate change is a long-term phenomenon and so for a long time has been studied as such. Climate models have looked at potential average changes over a space of decades and centuries. Socioeconomic and energy systems models have tried to project the effect of these changes in socio-technoeconomic systems. This study of trends over long time periods is how we've come to get a grasp on the scale and enormity of the phenomenon.

However, the long-term transformations are just one part of climate change. The other important aspect is the increase in the frequency and intensity of extreme weather events. This increase in extremes may be the aspect of climate change that most acutely affects human beings and the systems of our civilization, in particular energy systems. Many of the components of our energy system - from energy finance to liability allocation in under law - may not just be unprepared for extremes but in certain cases may exacerbate the impacts of extreme events.

Energy systems studies are not adequately equipped to analyse, predict or mitigate the risks of these extremes. The objective of this Focus issue is to highlight some of the blind spots concerning extremes in our knowledge of energy systems and to explore how extremes can be more systematically studied in different disciplines of energy science.

Extreme events can take many forms and can be borne of both natural and manmade systems. Consider for instance an anomalously hot day where the temperature is $2{ }^{\circ} \mathrm{C}$ higher than an average of, say, $35^{\circ} \mathrm{C}$ for that time of year. This doesn't appear to be a significant change in percentage terms, but if it is summer in a city where air conditioning is common, this change can lead to such a high and sudden jump in electricity demand for cooling that it can stress the grid to the point of failure (if there isn't enough back-up). This is what Perera et al. demonstrate in their Article by modelling energy demand separately for average and extreme temperature changes from climate models. As extremes cascade through systems, their effects magnify.

One particular magnifying force in man-made systems is herd behaviour in finance. Decision making in finance is driven by expectations of future growth or losses and such expectations have a natural tendency to become self-fulfilling prophecies. This is partly what's happening in the US coal industry. As cheaper natural gas floods the market, and fears of looming climate legislation spread, coal is seen as increasingly untenable in the future. As a result, finance is drying up for coal companies and the industry is shrivelling. On the other hand, renewable power may not be experiencing the kind of superexponential growth that's needed partly because it continues to be seen by the financial sector as not prone to explosive growth. In her Comment, Amy Jaffe explores the perils of this phenomenon and provides suggestions for how to curb it.

In general, as Paul Griffin notes in his Comment, the market may be only now starting to become somewhat aware of physical climate risks to firms. Challenges remain, however, towards accurate and complete pricing of risks, ranging from lack of data availability to a lack of adequate mathematical tools.

Indeed, extremes are difficult to model mathematically. What makes them striking and significant is the temporal and spatial specificity of their impact. This specificity, where and when and with what intensity an extreme event will strike, is impossible to predict with any long-term accuracy due to the chaotic nature of humanclimate systems.

Integrated climate-economy modellers have long struggled with this problem as David McCollum and colleagues note in their Comment. But as computing power and data availability grows and we begin to understand better the intersection of climate, energy and economic systems, we should do more to attempt to quantify at least how extremes, and not just average climate change, will affect energy systems. McCollum et al. take the first steps towards incorporation of extremes in energy systems models by building a typology of extremes.

Another way forward is suggested by Christian Otto and colleagues in their Comment. They propose we use our existing knowledge of the impacts of past extremes on the economy and energy systems, as well as projections of probable extreme events, to determine at least the scale of the imprint extremes can leave on 'business as usual'. Ideas such as those presented by McCollum and Otto and their respective colleagues provide a more systematic understanding of the ways in which extremes impact energy systems and the scale of their impacts.

We must also ensure that the projections and information we do have are presented to stakeholders in a manner that best supports their preparedness. For instance, in their Comment, Jana Sillmann and colleagues see seasonal extreme projections, such as forecasts for low precipitation summers or high wind winters, as already of benefit to the renewable energy industry, if its presentation suits the information needs of decision makers.

By and large though, the true impact of extremes continues to evade systematic study. As a result, the risk from extremes builds up without being accounted for. Sometimes this risk takes the form of legal liability to those potentially harmed by the extreme events. Justin Gundlach shows in his Comment how legal judgments may at times make the latent risk of extremes suddenly manifest, as in the case of courts in California holding the electric utility the Pacific Gas and Electric Company (PG\&E) strictly liable for the harm they caused, even just through locating their equipment in wildfire prone areas. In the future, the law may compel companies to take extreme risks more explicitly into account, but for now, the perception of risks from legal retribution and potential physical damage from extremes remains low and largely unaccounted for.

Accounting for and formalizing the impact of extremes is significant not just because it is the extremes that will break us but because the extremes affect the most vulnerable first and most devastatingly. Our awareness of the significant and disproportionate impact of extremes is increasing and the efforts to study them systematically have sped up. Nonetheless, much work remains in the areas of modelling, finance, legal and engineering analysis to understand and prepare for extreme events.

Published online: 19 February 2020 https://doi.org/10.1038/s41560-020-0572-2 